\title{
Laboratorios virtuales de electrónica básica para alumnos universitarios dentro del aula extendida
}

\author{
Virtual laboratories of basic electronics for university students within \\ the extended classroom
}

\section{Leonardo Navarria}

https://orcid.org/0000-0002-1061-4896

navarrial@fcaglp.unlp.edu.ar

Facultad de Ciencias Astronómicas y Geofísicas | Facultad de Ingeniería | Universidad Nacional de La Plata | Argentina

\author{
Alejandro González \\ https://orcid.org/0000-0002-9854-8618 \\ agonzalez@lidi.info.unlp.edu.ar \\ Instituto de Investigación en Informática LIDI \\ Facultad de Informática | Dirección General \\ de Educación a Distancia y Tecnologías | \\ Universidad Nacional de La Plata | Argentina
}

\author{
Alejandra Zangara \\ https://orcid.org/0000-0001-6013-9790 \\ alejandra.zangara@gmail.com \\ Instituto de Investigación en Informática III-LIDI | Facultad \\ de Informática | Universidad Nacional de La Plata | Argentina
}

\section{RESUMEN}

El objetivo de este trabajo es evaluar la efectividad que poseen las actividades prácticas de electrónica básica al realizarse con laboratorios virtuales dentro de un aula extendida. En la enseñanza presencial de carreras que poseen contenido práctico se hace fundamental la realización de prácticas para afianzar conocimientos. La ejercitación en las mismas involucra la asistencia del estudiante a determinados turnos de horarios fijos, en un lugar limitado en recursos de equipamiento y espacio. Con el uso de metodologías de trabajo virtuales se puede lograr que un gran número de alumnos tenga acceso a los contenidos prácticos para poder familiarizarse con instrumentos y componentes de electrónica básica que son de fundamental importancia para la adquisición de conocimientos prácticos. La pandemia COVID-19 ha fortalecido la realización de laboratorios virtuales.

\section{SUMMARY}

The purpose of this work is to evaluate the effectiveness of basic electronics practical activities with virtual laboratories within an extended classroom. In face-to-face teaching of careers that have practical content, it is essential to carry out practices to consolidate knowledge. Exercising in them involves the student's attendance at certain fixed-time shifts, in a place with limited resources for equipment and space. With the use of virtual work methodologies, it can be achieved that many students have access to practical content to become familiar with instruments and components of basic electronics that are of fundamental importance for the acquisition of practical knowledge. The COVID-19 pandemic has fostered the implementation of virtual laboratories.

PALABRAS CLAVE

prácticas, laboratorios, virtuales.

KEYWORDS

practical, laboratory, virtual. 


\section{1-INTRODUCCIÓN}

El presente trabajo deriva del trabajo de Tesis en curso para la obtención del Magister en Tecnología Informática Aplicada a la Educación dictada en la Facultad de Informática de la Universidad Nacional de La Plata.

El tesista es docente de la Facultad de Ingeniería y de la Facultad de Ciencias Astronómicas y Geofísicas, ambas de la UNLP. En las asignaturas que el autor ejerce su cargo docente se dictan contenidos básicos de electrotecnia, como ser leyes fundamentales, comportamiento de componentes eléctricos y electrónicos, análisis de distintos tipos de circuitos como ser en corriente continua como alterna. Por tratarse de materias de contenidos teóricos y prácticos, los análisis de los distintos tipos de circuitos pueden ser verificados utilizando simuladores de circuitos y también implementados de forma práctica, siendo aquí de vital importancia conocer el manejo de los instrumentos que permiten medir variables eléctricas.

En la enseñanza presencial de carreras que poseen contenido práctico se hace fundamental la realización de prácticas para afianzar conocimientos. La ejercitación en las mismas involucra la asistencia del estudiante a determinados turnos de horarios fijos, en un lugar limitado en recursos de equipamiento y espacio. Con el uso de metodologías de trabajo virtuales se puede lograr que un gran número de alumnos tenga acceso a los contenidos prácticos para poder familiarizarse con instrumentos y componentes de electrónica básica y avanzada.

La pandemia COVID-19 ha fortalecido la realización de laboratorios virtuales, ya que durante el 2020 y 2021 la Universidad Nacional de La Plata dictó de forma virtual todas las clases de las asignaturas en las cuales se estudiaron los laboratorios virtuales. 
Para evaluar el uso de los laboratorios se realizaron cuestionarios cerrados para alumnos y docentes, con el fin de conocer la percepción sobre el uso de los simuladores de clase. Con ello se buscó indagar la posibilidad de mejorar los contenidos, verificar si las prácticas de laboratorios mejoraron los procesos cognitivos, emplear simuladores en el proceso de enseñanza- aprendizaje y evaluar material informativo (Quintana; González; Vallejo; Pereyra (2019).

Para evaluar el uso de los laboratorios se realizaron cuestionarios cerrados para alumnos y docentes, con el fin de conocer la percepción sobre el uso de los simuladores de clase. Con ello se buscó indagar la posibilidad de mejorar los contenidos, verificar si las prácticas de laboratorios mejoraron los procesos cognitivos, emplear simuladores en el proceso de enseñanzaaprendizaje y evaluar material informativo

\section{2-PROPÓSITO}

\section{a) PRÁCTICA DE LABORATORIO}

La práctica de laboratorio es una estrategia pedagógica para la construcción de competencias procedimentales y por este motivo es utilizada en una gran variedad de programas académicos, usualmente articulada con su asignatura teórica correspondiente. El trabajo de Infante Jiménez (2014) aborda la importancia de los laboratorios presenciales en las carreras de ciencias aplicadas por ejemplo Ingeniería, Fisicoquímica, Química, etc., sin embargo, a medida que los modelos educativos se han vuelto más flexibles y enfocados a competencias, la inclusión de las Tecnologías de la Información y la Comunicación (TIC) ha cambiado radicalmente el concepto de espacio físico; esto ha mostrado una serie de limitaciones pues a pesar de la enorme importancia que éste tiene para el aprendizaje, no puede ofrecer la versatilidad idónea que se necesita en la actualidad. La práctica de laboratorio presenta elevados tiempos de respuesta, los cuales tienden a bajar su productividad.

Infante Jiménez (2014) remarca el costo de los experimentos con plantas reales respecto tiempo, dinero, energía, mantenimiento, condiciones que son difíciles de sostener si no se dispone de personal idóneo con alta disponibilidad frente a la demanda de las prácticas. También se debe tener en cuenta la supervisión por parte de docentes tutores o encargados de laboratorios. Todo esto conlleva a una limitación física de la cantidad de alumnos. Pensando en una época de pandemia COVID-19 
se le tendría que sumar el costo de la sanitización y que los laboratorios deberían ser de sólo un estudiante ya que por protocolo se imposibilita compartir elementos e instrumentos de medición.

El artículo de Contrera Gelves y otros (2010) explora los hábitos relacionados con el uso de simuladores para el apoyo en los procesos de transferencia del conocimiento en ciencias básicas y programación. En este trabajo se parte de la premisa para investigar el aporte de los simuladores a la formación de estudiantes universitarios de ingeniería. El estudio se contextualiza en el desarrollo de clases de los saberes de física, matemáticas y programación. Se contaron con laboratorios físicos dotados de puestos de trabajos suficientes para la cantidad de estudiantes.

La tarea del tutor consistirá en orientar y reorientar los procesos de comprensión y de mediar pedagógicamente a través de las actividades auto estructuradas y progresivas del estudiante a distancia.

Coincidiendo con el trabajo de Massa y Pesado (2012) se adopta la siguiente definición de objeto de aprendizaje: "la mínima estructura independiente que contiene un objetivo, un contenido, una actividad de aprendizaje, un metadato y un mecanismo de evaluación, el cual puede ser desarrollado con tecnologías de informática y comunicación (TIC) de manera de posibilitar su reutilización, interoperabilidad, accesibilidad y duración en el tiempo"

El laboratorio virtual como objeto de aprendizaje posee como atributos:

- Ser reutilizable: pueden volver a usarse sin perder ninguna característica.

- Ser digital.

- Ser un recurso: no constituye en sí mismo el conocimiento, sino que lo promueve y lo fortalece de manera más fácil.

- Servir de aprendizaje: hace hincapié a la característica de explícito e intencionado de que la persona debe aprender a través del objeto de aprendizaje

Como resultado "se puede decir, en forma general, que el uso de simuladores como estrategia didáctica, a través de los cuales se transfiere conocimiento, sí causa impacto en el proceso de aprendizaje de los estudiantes, ya que las clases se vuelven más interesantes, existe una mayor participación de los alumnos, son más claras las explicaciones que se dan, incrementan la retención al presentarse los contenidos, y aumenta la motivación y el gusto por aprender" (Contreras Gelves et al., 2010).

En la enseñanza de la ingeniería y carreras técnicas, es deseable la realización de actividades pedagógicas que le permitan al estudiante poner en práctica los conocimientos previos y adquirir nuevos, y 
poder corroborarse en el campo de la experiencia real. Este papel lo desempeña el laboratorio de prácticas, el cual, requiere de la presencia física del estudiante para poder manipular los sistemas de control y las plantas existentes en un entorno controlado, bajo la supervisión del profesor (Ibáñez y Moreno, 2018).

En la enseñanza de la ingeniería y carreras técnicas, es deseable la realización de actividades pedagógicas que le permitan al estudiante poner en práctica los conocimientos previos y adquirir nuevos, y poder corroborarse en el campo de la experiencia real.

\section{b) APRENDIZAJE BASADO EN PROBLEMAS}

El Aprendizaje Basado en Problemas (ABP) como metodología de enseñanza y de aprendizaje es utilizado en numerosas instituciones de educación superior en diversas áreas del conocimiento. La finalidad del ABP es formar estudiantes capaces de analizar y enfrentarse a los problemas de la misma manera en que lo hará durante su actividad profesional, valorando e integrando el saber que los conducirá a la adquisición de competencias profesionales (Araújo y Sastre, 2008).

La mejora de la resolución de situaciones utilizando ABP podrá favorecer en cuestiones fundamentales como ser:

- Unificación de criterios respecto a la resolución de problemas

- Unificación de lenguaje

- Resolución de situaciones

- Fundamentación correcta

- Adquisición de criterios para ejercer como futuro profesional.

Los trabajos de laboratorio, puestos en contexto de ABP y dependiendo del grado de participación del estudiante establece que:

- Se involucra al estudiante en el uso de procedimientos técnicos y científicos

- Se requiere metodología de trabajo

- Uso de material e instrumentos específicos, y en algunos casos reacondicionados y adaptados para que el estudiante los utilice para llevar adelante las prácticas.

- Se realizan en lugares distintos al aula dónde se dictan las clases, pudiendo ser estos un laboratorio, situando en el caso de estudio o de campo. 
- Se presentan riesgos para el alumno y daños en instrumentos respecto de la manipulación de algunos componentes.

- Se realizan actividades que poseen una complejidad de organización superior a la resolución de un ejercicio en el material de estudio.

- Se genera una motivación por parte del estudiante.

- Se ayuda a la comprensión de los contenidos teóricos expuestos en el aula.

- Se fomenta el desarrollo del razonamiento científico y problemas reales.

\section{DESARROLLO DEL LABORATORIO VIRTUAL DE ELECTRÓNICA BÁSICA}

Las transformaciones tecnológicas que permiten reducir la distancia han sido una de las causas del constante avance de la enseñanza no presencial.

La educación en la virtualidad, al igual que en la presencialidad existe la convivencia entre orientaciones y didácticas diversas, siempre que éstas actúen de forma coherente con las finalidades educativas y con los fines de la educación, de la misma forma sucede en la virtualidad (López Rua et al, 2012). El proceso de aprendizaje en ambientes virtuales es el resultado de varias etapas en las que el alumno construye su aprendizaje. Asimismo, puede ser el producto de la práctica, como puede ser el empleo de simuladores (Andrés; Pesa y Meneses, 2008).

A continuación, se listan algunos de los laboratorios virtuales realizados en el marco del desarrollo de la tesis:

\section{Análisis de circuitos en corriente continua:}

https://view.genial.ly/604a5ffecde1 db0d8262083e/learning-experience-didactic-unit-laboratorio-de-cc

\section{Análisis de circuitos en corriente alterna:}

https://youtu.be/Z6-fXSLZ1Pc

\section{Rectificadores:}

https://www.asignaturas.ing.unlp.edu.ar/mod/hvp/view. php?id=93774

https://youtu.be/k3vcU75SsUw

https://youtu.be/OKVOVrWAToU

https://youtu.be/SAEMP_zOwng

\section{Polarización de transistores:}

https://youtu.be/I1 gcED9iLSM 
Para la realización de los distintos laboratorios se utilizaron diferentes herramientas:

Laboratorio 1: se utilizó para el diseño web la herramienta de genial.ly (https://genial.ly/es), se utilizaron componentes físicos de circuitos eléctricos como ser protoboard, resistores, cables, etc. También se realizaron varias mediciones con instrumentos de medida de variables eléctricas para que el estudiante vincule el circuito real con un esquemático

Laboratorio 2: se utilizaron componentes físicos de circuitos eléctricos, resistores, cables, inductores y capacitores. Se utilizó un multímetro, osciloscopio y un generador de señales.

Laboratorio 3: para este laboratorio se utilizó un Branchscenario (H5P) la que permite ir tomando decisiones sobre el comportamiento de un determinado circuito. Asimismo, se utilizó un simulador de circuitos LTspice, junto al circuito real, instrumentos de medición y se filmó la realización de los laboratorios, comparando los resultados obtenidos con los simulados.

Laboratorio 4: Se implementó el circuito real y se utilizó un simulador de circuitos LTspice, junto a instrumentos de medición y se filmó la realización de los laboratorios, comparando los resultados obtenidos con los simulados.

A fin de no ocupar espacio en los servidores Universitarios se utilizó un canal de youtube para almacenar los laboratorios que al día 27 de julio han tenido un total de 576 visualizaciones, estando los videos configurados en "no listados", es decir, que solamente el alumnado posee el enlace de visualización.

Para poder diseñar los laboratorios el tiempo de edición, de medición, verificación y ajustes de estos debe tener la disponibilidad de los plazos para poder realizarlos y al mismo tiempo simularlos y filmarlos.

Los laboratorios en su mayoría han requerido un tiempo mínimo de cinco horas de realización contando con la edición y demás. Todas las filmaciones de los laboratorios fueron realizadas utilizando la herramienta OBStudio (https://obsproject.com/es), software libre y código abierto para grabación de video y transmisión en vivo, la misma corre bajo Windows, Mac o Linux. Los instrumentos utilizados, junto a los circuitos, fueron los disponibles en el laboratorio de Electrónica de la Facultad de Ciencias Astronómicas y Geofísicas y del ex Centro de Técnicas Analógicas y Digitales (CeTAD) del departamento de Electrotecnia de la Facultad de Ingeniería.

Cada uno de los laboratorios realizados fueron sometidos a juicio de expertos de cada una de las asignaturas correspondientes quienes realizaron una evaluación de estos sugiriendo mejoras y corrigiendo errores. 


\section{EVALUACIÓN DE LOS LABORATORIOS}

Para evaluar el impacto en los alumnos, se realizaron encuestas utilizando escala de Likert previas a comenzar con los primeros laboratorios y posteriores al último laboratorio.

\section{Para evaluar el impacto en los alumnos, se realizaron encues- tas utilizando escala de Likert previas a comenzar con los pri- meros laboratorios y posteriores al último laboratorio.}

La medición se realizó a través de indicadores como:

Utilidad: Es un indicador para estudiar dentro de la evaluación indirecta. Para elegir este indicador se centró en la idea del aprendizaje significativo. Este tipo de aprendizaje se caracteriza por el hecho de que el alumno vaya gestionando y edificando su aprendizaje como una escalera de tal forma que un escalón se basa en el otro. Para esto es muy importante que el alumno considere útil todo aquello que hace en el proceso educativo. Con ese indicador se buscó conocer el grado de utilidad que conceden los estudiantes a la formación recibida.

Uso del laboratorio: Se pretendió medir cual era la opinión de los estudiantes sobre la usabilidad del sistema implementado en el laboratorio remoto. Se planteó la fase previa y posterior al laboratorio lo que sirvió para recolectar información sobre lo esperado y lo obtenido de los estudiantes gracias a la implementación del laboratorio. Toda esta información aportó datos para planificar la formación y mejorar el aprendizaje de los alumnos a través de los laboratorios remotos.

Los objetivos específicos se centraron en:

1. Ampliar el alcance de los trabajos prácticos propuestos en las clases de explicación práctica

2. Profundizar el análisis de los problemas planteados, llevando las experiencias a mediciones de situaciones reales.

3. Analizar los indicadores de expectativas, estimulación, utilidad del laboratorio, uso del laboratorio remoto

4. Proponer orientaciones de uso del laboratorio virtual en el aula de electrónica

La muestra para poder llevar adelante el estudio fueron las asignaturas, en las cuales el tesista se desarrolla como docente en el primer semestre del año:

Cátedra Análisis de Circuitos, de la Carrera Ingeniería en Electró- 
nica, Electricista y en Telecomunicaciones de la Facultad de Ingeniería de la Universidad Nacional de La Plata. Cantidad de alumnos regulares de cursada: 15

Cátedra Dispositivos Electrónicos, de la Carrera Ingeniería en Electrónica, Electricista y en Telecomunicaciones de la Facultad de Ingeniería de la Universidad Nacional de La Plata. Cantidad de alumnos regulares de cursada: 35

Cátedra Instrumentos y Observación, perteneciente a la carrera Licenciado en Meteorología y Ciencias de la Atmósfera de la Facultad de Ciencias Astronómicas y Geofísicas de la Universidad Nacional de La Plata. Cantidad de alumnos regulares de cursada: 11

Cátedra Instrumental Geofísico y Electrónico, perteneciente a la carrera Geofísico de la Facultad de Ciencias Astronómicas y Geofísicas de la Universidad Nacional de La Plata. Cantidad de alumnos regulares de cursada: 18

Se implementaron encuestas cerradas utilizando formularios de Google en dónde se consultó sobre:

Expectativas: acerca del aumento de las habilidades, conocimientos, y formación del estudiante.

Utilidad: acerca de la mejora de competencias y capacidades para resolver circuitos eléctricos.

Contenido y método: acerca de la consolidación de conocimientos básicos, la ampliación de las herramientas utilizadas y si los contenidos didácticos fueron precisos, comprensibles y actualizados

Herramientas: aplicaciones multimedia, recursos informáticos, simuladores, adecuación respecto de los contenidos

Resultados de Encuestas Previas

Las encuestas previas fueron respondidas por un total de 68 alumnos.
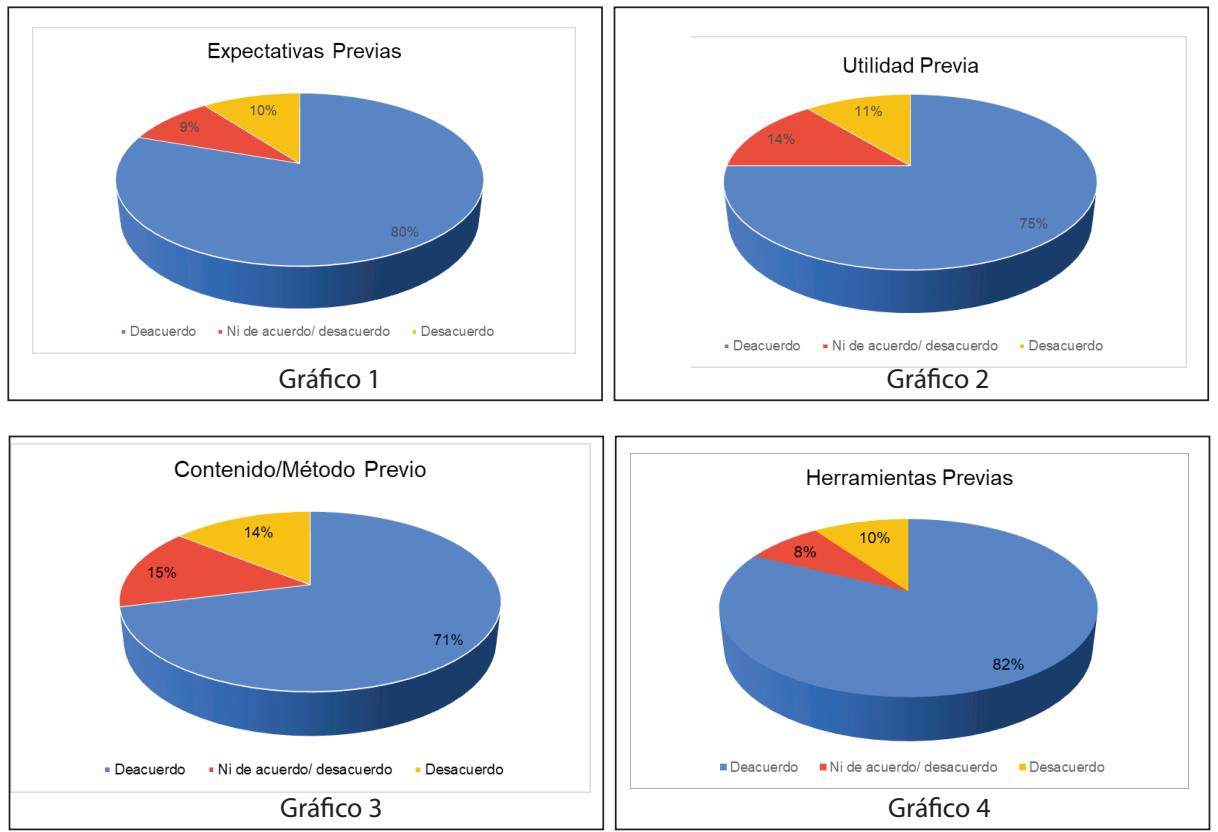

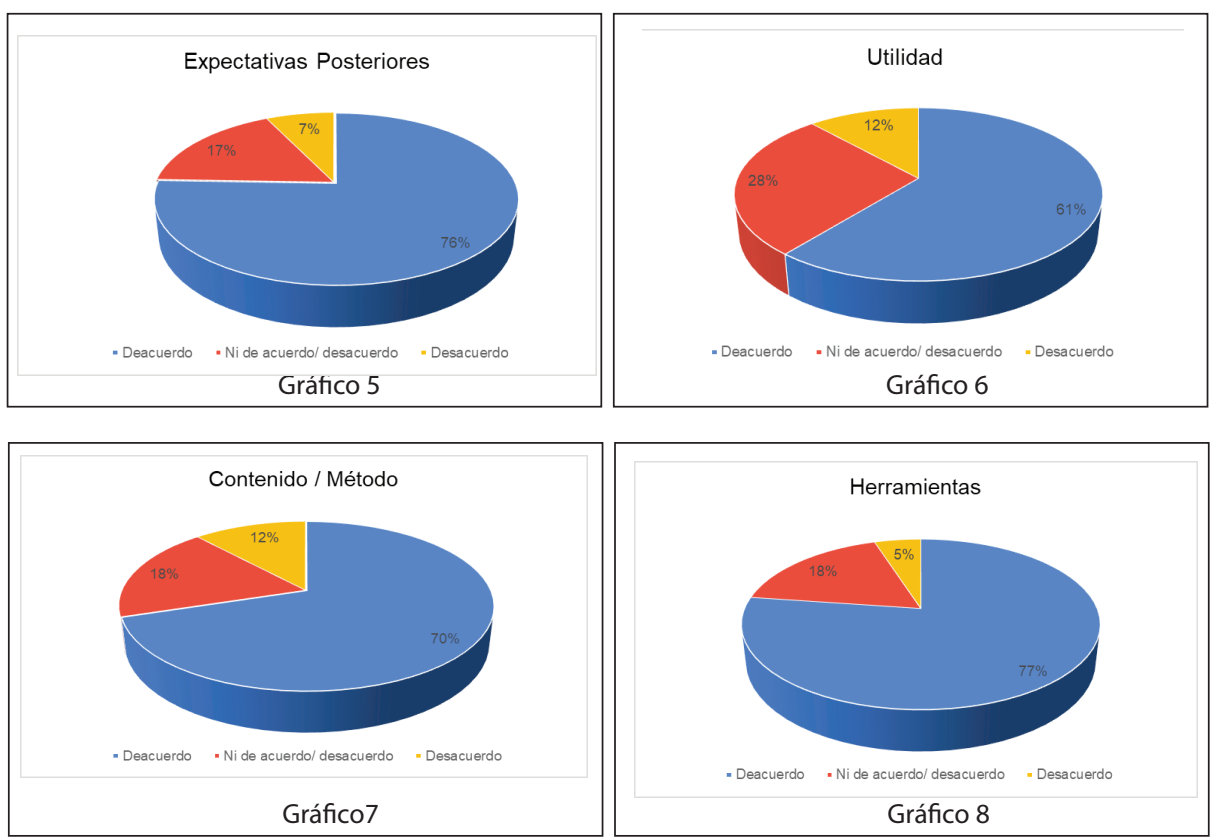

Respecto de las expectativas (gráficos 1 y 5) se puede afirmar que se han cumplido pudiendo afirmar que los laboratorios han mejorado los conocimientos.

Observando la utilidad (gráficos 2 y 6), se detecta que un porcentaje de los alumnos cree que no han sido tan útiles como pensaban. Aquí se debe rearticular que los trabajos prácticos presentados en las cátedras y los laboratorios deban estar acorde a sus contenidos.

Respecto a los contenidos (gráficos 3 y 7) y los métodos abordados en los laboratorios, no hay grandes cambios respecto a lo esperado y a la obtenido.

En cuanto a las herramientas (gráficos 4 y 8 ) multimedia utilizadas para realizar los laboratorios ha disminuido en pequeños porcentajes lo esperado de lo obtenido. Siendo el resultado aceptable.

\section{CONCLUSIONES}

La pandemia generada por el COVID-19 ha forzado a que se implemente la virtualidad como método de enseñanza en los niveles educativos comenzando desde inicial hasta cursos de posgrado. Los laboratorios desarrollados en este trabajo fueron evaluados por docentes expertos en las materias y por los alumnos que los utilizaron. El intenso trabajo en el desarrollo de los laboratorios, la especialización en herramientas de diseño, las ideas para poder implementarlos han generado como resultado obtenido de las encuestas de los alumnos un cumplimiento de las expectativas planteadas, teniendo la ventaja que todos los laboratorios realizados son reutilizables para futuros cursos. 


\section{BIBLIOGRAFÍA}

Andrés, M. M., Pesa, M. y Meneses, J. (2008). Efectividad de un laboratorio guiado por el modelo de aprendizaje matlab para el desarrollo conceptual asociado a tareas experimentales. Enseñanza de las Ciencias, 2008, 26(3), 343-358.

Araújo, U. F., y Sastre, G. (coords.) (2008). El Aprendizaje Basado en Problemas. Barcelona: Gedisa Editorial

Contreras Gelves, Gloria Amparo, \& García Torres, Rosa, \& Ramírez Montoya, María Soledad (2010). Uso de simuladores como recurso digital para la transferencia de conocimiento. Apertura, 2. ISSN: 1665-6180. Disponible en: https://dialnet.unirioja.es/servlet/articulo?codigo $=5547092$

Ibáñez, J. M. S., \& Moreno, J. B. A (2018) Uso de simuladores en el aula para favorecer la construcción de modelos mentales. Edutec Chile 2018.

Jiménez, C. I. (2014). Propuesta pedagógica para el uso de laboratorios virtuales como actividad complementaria en las asignaturas teórico-prácticas. Revista mexicana de investigación educativa, 917-937. Obtenido de http://www.scielo.org.mx/scielo.php?script=sci_arttext\&pid=S1405-66662014000300013\&In$\mathrm{g}=\mathrm{es} \& \operatorname{tn} \mathrm{n}=\mathrm{es}$

López Rua, Ana Milena, \& Tamayo Alzate, Óscar Eugenio (2012). Las Prácticas De Laboratorio En La Enseñanza De Las Ciencias Naturales. Revista Latinoamericana de Estudios Educativos (Colombia), 8(1),145-166. ISSN: 1900-9895. Disponible en: https://www.redalyc.org/articulo.oa?id=134129256008.

Massa, S. M., \& Pesado, P. M. (2012). Evaluación de la usabilidad de un Objeto de Aprendizaje por estudiantes. TE \& ET. Disponible en: http://sedici.unlp.edu. ar/handle/10915/25537

Quintana, N., González, A. H., Vallejo, A. E., \& Pereyra, J. M. (2019). Simulador en línea para capacitación de individuos hipoacúsicos adultos. In XXV Congreso Argentino de Ciencias de la Computación (CACIC 2019, Universidad Nacional de Río Cuarto). 